\title{
Millimeter VLBI observations: Black Hole Physics and the Origin of Jets
}

\author{
T.P. Krichbaum*, A. Roy, R.-S. Lu, J.A. Zensus \\ Max-Planck-Institut für Radioastronomie, 53121 Bonn, Germany \\ E-mail: tkrichbaumempifr.de
}

\section{Fish, S. Doeleman}

MIT Haystack Observatory, Westford, MA 01886, USA

\section{on behalf of the Event Horizon Telescope (EHT) collaboration}

\begin{abstract}
VLBI observations at the highest possible frequency can overcome the opacity barrier in the nuclear regions of radio-galaxies and blazars, which are synchrotron self-absorbed at longer wavelength. This facilitates a direct and sharp view into the 'heart' of Active Galactic Nuclei (AGN), into regions in which $\mathrm{BH}$ physics and general relativity become important and radio jets are launched. Here we report on new results from global $3 \mathrm{~mm}$ and $1.3 \mathrm{~mm}$ VLBI observations. In M 87, the $3 \mathrm{~mm}$ GMVA data reveal a conical jet with jet opening of $\sim 60^{\circ}$ on $\leq 25$ Schwarzschild radii scales emerging from a compact core. Faint emission on the counter-jet side suggests a jet-to-counter-jet ratio of $R \sim 10-25$. The $1.3 \mathrm{~mm}$ VLBI imaging - now including the APEX telescope - reveals ultra-compact structures in Sgr A* and M 87, corresponding to emission regions of $\sim 3 R_{S}$ in size. A first $230 \mathrm{GHz}$ VLBI image of BL Lac shows a one-sided north-south oriented core-jet structure with an unresolved $(\leq 25 \mu \mathrm{as})$ VLBI core of high brightness temperature $\left(\geq 10^{11} \mathrm{~K}\right)$. The planned participation of the phased ALMA in VLBI will boost the imaging capabilities of mm-VLBI and hopefully soon will lead to the direct imaging of nearby Back Holes.
\end{abstract}

12th European VLBI Network Symposium and Users Meeting,

7-10 October 2014

Cagliari, Italy

${ }^{*}$ Speaker. 


\section{Introduction and Motivation}

The extreme luminosities observed in many active galactic nuclei (AGN: Blazars, Radio Galaxies) is commonly explained by matter accretion onto a central super massive black hole (SMBH). The physical details of this gravitational energy conversion into kinetic outflow and radiation are still not well understood, and it is now discussed whether electro-magnetic processes are playing a more important role than previously assumed. For a realistic judgment of the relative importance of mechanical, gas-kinematic, radiation and electro-magnetic processes acting near the BH, good observational data are needed as a constraint. This is also needed for the modern computer simulations, which now take general relativistic dynamo-effects (GRMHD) into account. The very efficient launching of powerful relativistic radio jets explains about $10-15 \%$ of the total population of galaxies, the so called radio-loud emitting AGN population. However, it is not very clear why the larger majority of the other galaxies remain radio-quiet (or better radio-faint), and whether this is related to specific physical conditions in the black hole accretion disk system, such as the presence of high/low accretion rates, thick/thin disks or just by the strength/orientation of the $\mathrm{BH}$ spin.

Very Long Baseline Interferometry (VLBI) at short millimeter and sub-millimeter wavelength provides an angular resolution of $\sim 25 \mu$ as (at $230 \mathrm{GHz}$ ), small enough to probe the region of jet-formation in AGN and at frequencies, where synchrotron self-absorption and internal opacity decrease. Perhaps even more important for nearby SMBHs, the resolution offered by mm-/submmVLBI is high enough to image their surrounding emission regions, eventually even the BH shadow directly, if the SMBH is surrounded by millimeter-wave emitting matter. The detection of an 'Event Horizon' signature would not only prove the existence of a black hole, but also offer the amazing option to study and test General Relativity in the regime of strong gravity.

\section{Global 3 mm VLBI}

The Global millimeter-VLBI Array (GMVA) primarily observes at $86 \mathrm{GHz}$, combining eight VLBA stations equipped with receivers (SC, HN do not have $3 \mathrm{~mm}$ ), with the following European stations: $100 \mathrm{~m}$ Effelsberg, $30 \mathrm{~m}$ IRAM Pico Veleta, 6 x $15 \mathrm{~m}$ IRAM Plateau de Bure, $40 \mathrm{~m}$ Yebes, $20 \mathrm{~m}$ Onsala, and $14 \mathrm{~m}$ Metsähovi. This yields an angular resolution of up to $45 \mu$ as, allowing high spatial resolution (sub-pc scale) imaging of compact radio sources [1,2]. The GMVA is open to the scientific community and accepts observing proposals at submission deadlines, which are synchronized with the NRAO (Feb. 1st \& Aug. 1st each year). Purely for logistical reasons, GMVA observations are usually performed in up to 5 day long observing sessions (block observing the approved projects) twice per year in Spring and Autumn.

In Figure 1 we show as example a $86 \mathrm{GHz}$ GMVA map of the nearby radio galaxy M87 ( $D=$ $16.7 \mathrm{Mpc}, 1 \mathrm{mas}=0.081 \mathrm{pc}$ ) observed in May 2009. The image is based on two full uv-coverages observed on two adjacent days. Reasonable weather and the participation of the IRAM telescopes allowed to make a good quality image with a dynamic range of $\sim 330$. The map shows a conical core jet structure, which is also seen at longer wavelength and larger core separations [5]. The data also show evidence for the presence of a counter-jet, the first time in an $86 \mathrm{GHz}$ VLBI image. This is consistent with previous counter-jet detections at longer wavelength [3, 4], but needs confirmation 

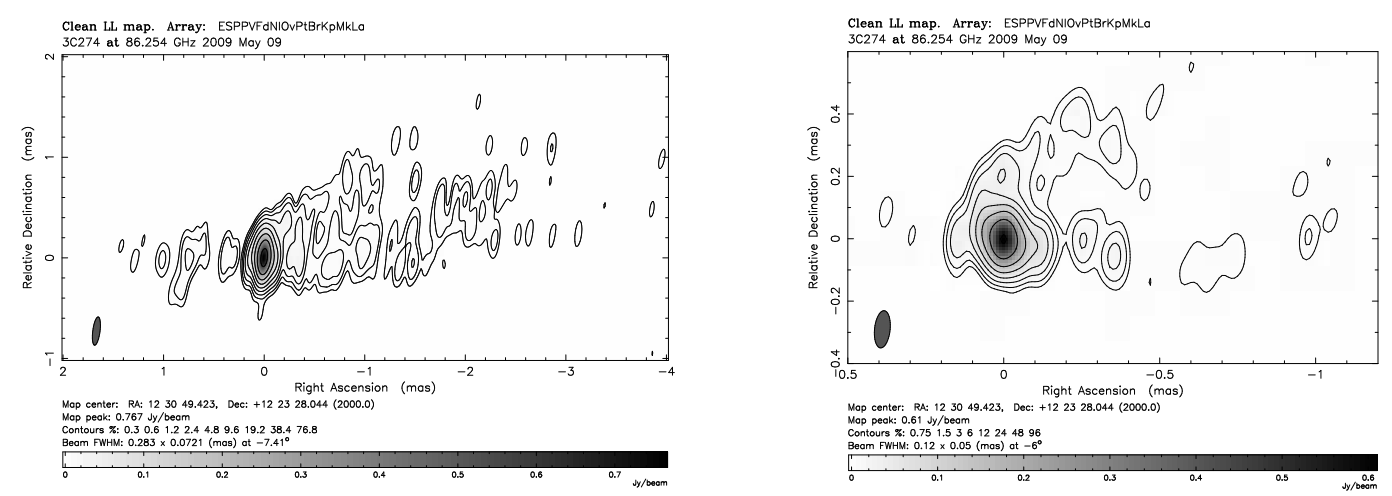

Figure 1: M87 observed with the GMVA at $86 \mathrm{GHz}$ in May, 2009. Left: Clean map convolved with a tapered beam (uv-taper 0.1 at $3 \mathrm{G} \lambda$ ). Right: The same map not tapered and convolved with a smaller beam, which has a factor of 2 super-resolution in north-south direction.

by higher sensitivity imaging. (see below). Convolving the map with a more circular beam (Fig. 1, right panel) shows a filamentary structure extending west and north-west to the core. This can be interpreted as part of the edge-brightened and transversely resolved inner jet cone, which extends at an opening angle of $\sim 60^{\circ}$ on scales of $\leq 0.2$ mas or $\leq 25$ Schwarzschild radii.

The availability of new digital backends (DBBC, RDBE) allows to perform VLBI observations at a larger data rate of $2 \mathrm{Gbps}$ and increased bandwidth ( $256 \mathrm{MHz}$ per polarization). This pushes the formal sensitivity of the full GMVA array to $\sim 1 \mathrm{mJy} / \mathrm{hr}$. Due to its larger collecting area (more big telescopes) the GMVA is about twice as sensitive as the VLBA ( $2.5 \mathrm{mJy} / \mathrm{hr}$ for 8 stations). It also offers an about $2-3$ times higher angular resolution due to its better sensitivity on the very longest baselines. In order to further improve the sensitivity at $86 \mathrm{GHz}$, VLBI observations including the $100 \mathrm{~m}$ Green Bank Telescope (GBT) were made in February 2013. The GBT performed well with a typical aperture efficiency of $\eta_{A}=0.26$ at $86 \mathrm{GHz}$. Participation of the GBT will almost double the array sensitivity $(0.5 \mathrm{mJy} / \mathrm{hr}$ for GMVA+GBT, $1.0 \mathrm{mJy} / \mathrm{hr}$ for VLBA+GBT). For the near future it is also foreseen that the three telescopes from the Korean VLBI Network (KVN) participate in global $3 \mathrm{~mm}$ VLBI. Pilot experiments performed in 2012 and 2013, have already revealed $86 \mathrm{GHz}$ fringe detections between KVN and Europe with good phase stability, eg. between the KVN and the phased Plateau de Bure $(0716+714$, SNR $~ 20)$. We note a present bandwidth limitation of the KVN in VLBI recording to $1 \mathrm{Gbps}$. The addition of telescopes in East-Asia will considerably improve the uv-coverage, particularly at those times, when a source is above the horizon in Europe, but not yet in America.

\section{Global 1mm VLBI, preliminary results from March 2013}

After the successful detection of VLBI fringes at $230 \mathrm{GHz}$ between two US-stations and the APEX telescope in Chile in 2012 [6], a global observing campaign with stations at 5 different sites (PV, SMTO, CA, SMA, JCMT, AP, see Table 1) was performed in March 2013. Within a pre-allocated observing window of 10 days, VLBI observations were triggered on 6 different days 


\begin{tabular}{|c|c|c|c|c|c|c|c|c|c|}
\hline \multirow[b]{2}{*}{ Antenna } & \multirow[b]{2}{*}{ Id } & \multicolumn{6}{|c|}{ Table 1: The mm-VLBI Array } & \multirow[b]{2}{*}{ Pol. } & \multirow[b]{2}{*}{ comment } \\
\hline & & $\begin{array}{c}\mathrm{D}_{e f f} . \\
{[\mathrm{m}]}\end{array}$ & $\begin{array}{c}\text { Surface } \\
{[\mu \mathrm{m}]}\end{array}$ & Eff. & $\begin{array}{l}\text { Gain } \\
{[\mathrm{K} / \mathrm{Jy}]}\end{array}$ & $\begin{array}{l}\text { Tsys } \\
{[\mathrm{K}]}\end{array}$ & $\begin{array}{c}\text { SEFD } \\
\text { [Jy] }\end{array}$ & & \\
\hline Pico Veleta & PV & 30 & 67 & 0.43 & 0.110 & 250 & 2273 & dual & \\
\hline CARMA & $\mathrm{CA}$ & 30.8 & $60 / 29$ & 0.55 & 0.148 & 250 & 1689 & dual & $6 \times 10.4 \mathrm{~m}+8 \times 6.1 \mathrm{~m}$ \\
\hline Submm-Array & SMA & 16.1 & 12 & 0.70 & 0.051 & 150 & 2941 & LCP & $8 \times 6 \mathrm{~m}$ \\
\hline J.Clerk Maxwell & JCMT & 15 & 24 & 0.63 & 0.040 & 350 & 8750 & $\mathrm{RCP}$ & \\
\hline APEX & $\mathrm{AP}$ & 12 & 18 & 0.63 & 0.026 & 350 & 13462 & LCP & \\
\hline Heinrich-Hertz & SMTO & 10 & 15 & 0.64 & 0.018 & 240 & 13333 & dual & \\
\hline South Pole & SPT & 10 & 20 & 0.58 & 0.016 & 200 & 12500 & dual & \\
\hline Large Millimeter & LMT & 32 & 75 & 0.30 & 0.086 & 250 & 2900 & dual & $32 \mathrm{~m}$ illumination \\
\hline ALMA & ALMA & 85 & 25 & 0.66 & 1.36 & 150 & 110 & dual & $53 \times 12 \mathrm{~m}$ \\
\hline Plateau de Bure ${ }^{1}$ & PdBI & 34.9 & 55 & 0.45 & 0.156 & 250 & 6410 & dual & $6 \times 15 \mathrm{~m}$ \\
\hline Greenland & GLT & 12 & 47 & 0.57 & 0.023 & 150 & 6400 & dual & under construction \\
\hline
\end{tabular}

${ }^{1}$ : PdBI will be upgraded to NOEMA, doubling antenna number and collecting area.

between March 20-27, 2013. The data were recorded in MK5 B format, using the RDBE/DBBC2 backends at a data rate of $3.8 \mathrm{Gbps}(15 \times 32 \mathrm{MHz}, \mathrm{RCP} \& \mathrm{LCP}$, tuning in Nyquist zone $2 \& 3)$. The ongoing data analysis reveals strong $(S N R \leq 250)$ fringes on most baselines of the array and for many AGN. Because of its receiver, the APEX telescope recorded only one polarization (LCP). On the baselines to APEX the following sources are detected so far: 3C 273 (23), M87 (11), 3C279 (16), 1749+096 (31), Sgr A* (11), 1633+38 (30), 1921-293 (10), 2013+370 (20), BL Lac (115), where the number in brackets denote the maximum SNR, achieved in either of the two Nyquist zone (each of $480 \mathrm{MHz}$ bandwidth). During this VLBI campaign, the $30 \mathrm{~m}$ IRAM telescope at Pico Veleta suffered from weather, which led to a time limited participation in VLBI. Still, clear fringe detections between Pico Veleta, APEX and the three US sites were obtained on March 25. In particular we note the detection of BL Lac on the long PV-AP baseline (SNR $\leq 15$ at $6.4 \mathrm{G} \lambda$ ) and faint detections on the even longer PV-SMA baseline (SNR $\leq 7$ at $8.3 \mathrm{G} \lambda$ ). These results not only indicate the technical feasibility of $1.3 \mathrm{~mm}$ VLBI even on the longest baselines (SpainHawaii, Spain-Chile), but also proofs the existence of ultra-compact structures in AGN, with sizes of typically less than $(25-30) \mu$ as. We also note that the SNR of the detections on the longest baselines not necessarily correlates with the total flux of the source, but depends on an intrinsic compactness, which could be high also in fainter sources.

The $230 \mathrm{GHz}$ fringe detections of Sgr A* (Fig. 2) and M 87 (Fig. 3) on APEX baselines result in Gaussian sizes (FWHM) in the range of 25-35 $\mu$ as, corresponding to about $2.5-3.5$ gravitational radii for each source, which is somewhat smaller than previously reported [7, 8]. We note, however, that these (preliminary) size estimates depend on the details of the amplitude calibration (Tsys, station gain), which at present is uncertain by $\sim 20-30 \%$. The orientation of the VLBI baselines to APEX is significantly different and more north-south oriented, than the mainly east-west oriented baselines of the North-American sub-array (Fig. $2 \& 3$, left panel). Small sizes derived from the visibilities to APEX therefore may indicate a non-circular source structure, i.e. an elliptical or more complex brightness distribution. Despite the sparse uv-coverage and very limited closure-phase information, it is possible to fit simple Gaussian models to the visibilities. 

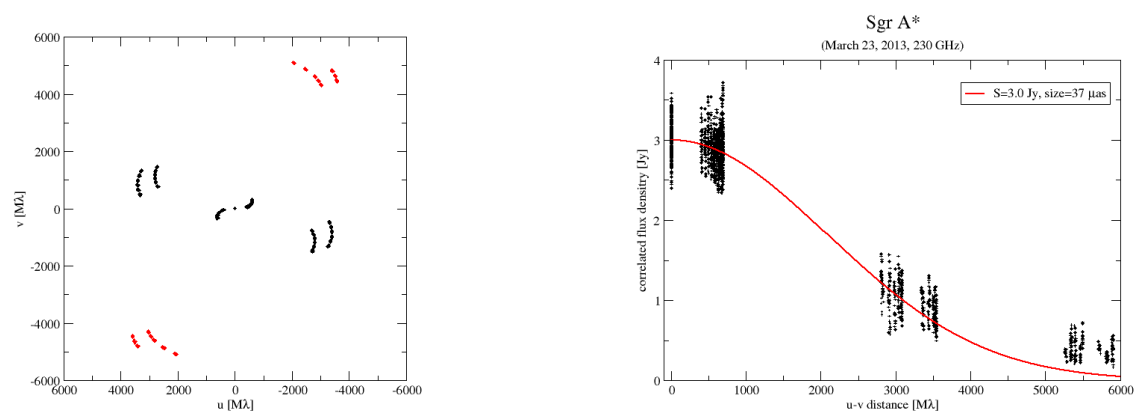

Figure 2: Left: uv-coverage for Sgr A* at $230 \mathrm{GHz}$ on March 23, 2013. The fringing baselines to APEX are marked red. Right: Correlated flux vs. projected baseline length for Sgr A* at $230 \mathrm{GHz}$. The data from APEX are at $>5 \mathrm{M} \lambda$. The red line results from a fit of a circular Gaussian component to the uv-data of the US-stations only (uv-range: $0-4 \mathrm{M} \lambda$ ).
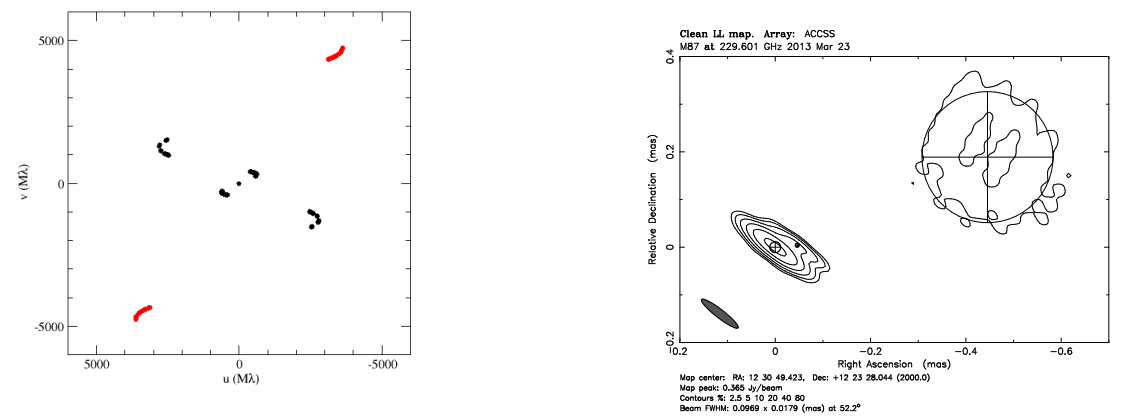

Figure 3: Left: uv-plot for M87 at $230 \mathrm{GHz}$ in March 2013, the fringing APEX baseline is marked in red. Right: A preliminary three component Gaussian model, which fits the $1.3 \mathrm{~mm}$ VLBI data.

For M 87, one possible representation of its sub-mas structure is shown in Fig. 3 (right panel). The model shows a core-jet like extension to the west, consistent with the overall source orientation seen with VLBI at longer wavelengths (see the $86 \mathrm{GHz}$ image, Fig. 2), and indicating the presence of a compact jet base with a brightness temperature of $T_{B}>10^{10} \mathrm{~K}$.

Figure 4 (center) shows a $1.3 \mathrm{~mm}$ VLBI map of BLLac, which was obtained from observations on March 27 using 5 VLBI stations (SMTO, SMA, CARMA-phased, CARMA-single, APEX, see Tab. 1). The uv-coverage for this image is shown in Figure 4 (left). Despite this limited uvcoverage, the basic source structure seen at $1.3 \mathrm{~mm}$ is remarkably similar to a $3 \mathrm{~mm}$ VLBI map, obtained with the VLBA on Feb. 18, 2013 (Fig. 4, right). In the VLBI imaging at $230 \mathrm{GHz}$, the available very short baseline between a single $10.8 \mathrm{~m}$ CARMA antenna and the phased CARMA array helped a lot to better constrain the total source flux, which in data sets without such a short baseline could not be fully recovered. Applying uniform weighting and no uv-tapering yields a beam size of $(24 \times 60) \mu$ as, which translates at the distance of BLLac $(z=0.0686)$ to a spatial scale of $(3 \times 7.5) 10^{-2} \mathrm{pc}$, where the minor axis of the beam corresponds to $\sim 300$ Schwarzschild radii for a $10^{9} M_{\odot}$ BH. A Gaussian model-fit to the central structure yields a core flux of $\sim 5.3 \mathrm{Jy}$ and a size of $\leq 20 \mu$ as which corresponds to a linear scale of $\leq 200$ Schwarzschild radii. The brightness 

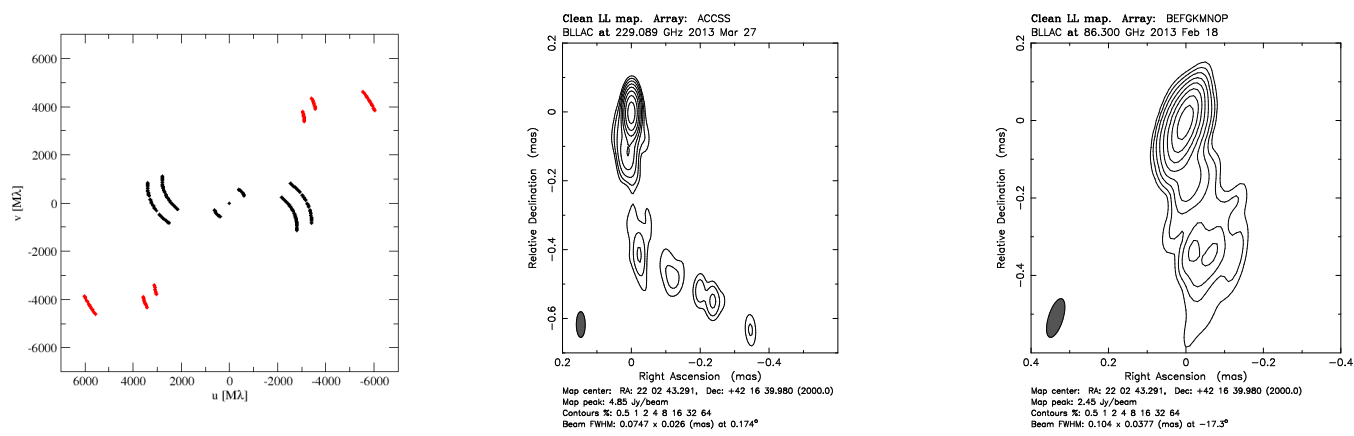

Figure 4: Left: uv-plot for BL LAC at $230 \mathrm{GHz}$ in March 2013, the fringing APEX baselines are marked in red. Center: $1.3 \mathrm{~mm}$ VLBI map of BL Lac, in a CLEAN map displayed with 0.3 uv-tapering at $7 \mathrm{G} \lambda$. Right: $3 \mathrm{~mm}$ VLBI image observed near in time with the VLBA.

temperature of the core is therefore a $T_{B} \geq 10^{11} \mathrm{~K}$. This is below the $10^{12} \mathrm{~K}$ inverse Compton limit, but higher than typical brightness temperatures seen in other sources observed at $230 \mathrm{GHz}$. The high brightness temperature is likely related to the fact, that BL Lac was still very compact and bright after a major submm-flare, which peaked mid November 2012 ( $S \simeq 14 \mathrm{Jy}$ at $340 \mathrm{GHz}$ ).

\section{Summary and Outlook}

During the last few years the capabilities of global mm-VLBI have been continously improved, adding more telescopes and sensitivity to the arrays at $3 \mathrm{~mm}$ and $1.3 \mathrm{~mm}$, This allows imaging with the highest resolution of many more and fainter objects. The technical development of the digital VLBI recording systems aims at an instantaneous observing bandwidth of $8 \mathrm{GHz}$ ( $32 \mathrm{Gbps}$ data rate), which is covered by the ALMA (band $3 \& 6$ ) receivers. In the very near future VLBI phasing capability will become available for ALMA, both at $3 \mathrm{~mm}$ and $1.3 \mathrm{~mm}$. This will mark a transition in global mm-VLBI and its scientific capabilities.

\section{References}

[1] J. Hodgson, et al., 2014, 5 year Global 3-mm VLBI survey of Gamma-ray active blazars, in Proceedings of 12th EVN Symposium, PoS (EVN 2014) 028.

[2] V. Karamanavis, et al., 2014, Localizing the $\gamma$ rays from blazar PKS 1502+106, in Proceedings of 12th EVN Symposium, PoS (EVN 2014) 087.

[3] C. Ly, R. C. Walker, \& W. Junor, 2007, High-frequency VLBI imaging of the jet base of M 87, ApJ 660, 200.

[4] Y.Y. Kovalev, M.L. Lister, D.C. Homan, \& K.I. Kellermann, 2007, The inner jet of the radio galaxy M 87, ApJ 668, L27.
[5] M. Nakamura \& K. Asada, 2013, The parabolic jet structure in M87, ApJ 775, 118.

[6] A.L. Roy et al., 2012, VLBI at APEX: First Fringes, in Proceedings of 11th EVN Symposium, PoS (11th EVN Symposium) 057.

[7] V.L. Fish et al., 2011, $1.3 \mathrm{~mm}$ Wavelength VLBI of Sgr A*: Detection of Time-variable Emission on Event Horizon Scales, ApJ 727, 36.

[8] S.S. Doeleman et al., 2012, Jet-Launching Structure Resolved Near the Supermassive Black Hole in M87, Sci 338, 355. 